\title{
Comorbidity indices for clinical trials: adaptation of two existing indices for use with the FREEDOM trial in women with postmenopausal osteoporosis
}

\author{
S. L. Silverman ${ }^{1,5}$ - A. Wang ${ }^{2}$ L. Cheng ${ }^{2} \cdot$ Y. Yang $^{2}$ - C. Libanati ${ }^{2} \cdot$ M. Geller $^{2}$ • \\ A. Grauer ${ }^{2} \cdot$ M. Nevitt ${ }^{3}$ D. Revicki ${ }^{4} \cdot$ H. N. Viswanathan ${ }^{2}$
}

Received: 26 March 2015 / Accepted: 15 June 2015 /Published online: 15 July 2015

(C) The Author(s) 2015. This article is published with open access at Springerlink.com

\begin{abstract}
Summary Two comorbidity indices were adapted for use in the FREEDOM trial and significantly correlated with the number of medications and impaired health status at baseline. The indices have applications for the analysis of clinical trial data and would allow for the appropriate adjustment of comorbidities when evaluating clinical trial outcomes.

Introduction The purpose of this study is to adapt two published comorbidity indices for use with the FREEDOM clinical trial evaluating postmenopausal women with osteoporosis.

Methods FREEDOM enrolled women aged 60-90 years with a bone mineral density T-score $<-2.5$ at the lumbar spine or total hip and $\geq-4.0$ at both sites. Comorbidity indices were calculated using methods described by Sangha (Arthritis Rheum 49:156163,2003 ) and Wolfe (J Rheumatol 37:305-315, 2010) following modification. The adapted Sangha index included 12 conditions with a summary score of $0-12$; the adapted Wolfe index included 7 conditions with a weighted summary score of $0-8$. Higher scores indicated greater comorbidity. A panel of clinicians independently reviewed subjects' medical histories using
\end{abstract}

Electronic supplementary material The online version of this article (doi:10.1007/s00198-015-3215-x) contains supplementary material, which is available to authorized users.

S. L. Silverman

stuarts@bhillsra.com

1 UCLA School of Medicine, and OMC Clinical Research Center, Cedars-Sinai Medical Center, Los Angeles, CA, USA

2 Amgen Inc., Thousand Oaks, CA, USA

3 University of California San Francisco, San Francisco, CA, USA

4 Evidera, Bethesda, MD, USA

58641 Wilshire Blvd, Suite 301, Beverly Hills, CA 90211, USA a systematic process based on Medical Dictionary for Regulatory Activities (MedDRA) preferred terms to map specified comorbid conditions. Spearman correlations between the adapted indices and baseline subject characteristics expected to be associated with comorbidities were examined.

Results Of the 7808 subjects in this study, $74 \%$ had $\geq 1$ comorbidities based on the adapted Sangha or Wolfe comorbidity indices. The mean (SD) adapted Sangha and Wolfe comorbidity indices were 1.4 (1.2) and 1.4 (1.3), respectively. Both indices correlated positively with age, body mass index, and the number of medications ( $r=0.54$ to 0.55$)$ at baseline and inversely correlated with health-related quality of life ( $r=$ -0.22 to -0.30 ) (all $P<0.0001)$. Further, when either the adapted Sangha or Wolfe index was included as a covariate for assessing mortality over 36 months in the FREEDOM population, the hazard ratio of the comorbidity index indicated that the mortality risk increased by 27 or $28 \%$, respectively, for each unit increase in the adapted index (both $P<0.0001$ ). Conclusions Our work suggests these comorbidity indices may be adapted for use with clinical trial data, thereby allowing for the appropriate adjustment and reporting of covariates in the evaluation of clinical trial outcomes in an osteoporotic population.

Keywords Comorbidity $\cdot$ Index $\cdot$ Osteoporosis $\cdot$ Sangha index $\cdot$ Wolfe index

\section{Introduction}

The presence or absence of comorbid conditions and the number of such conditions are important factors in interpreting clinical trial results. In secondary analyses of pooled clinical trial data designed to answer specific research questions, adjustment for patients' comorbidity status can provide critical insights and may have an impact on the interpretation of the data. Although 
medical history is collected in clinical trials, the information collected does not lend itself to the calculation of a comorbidity index. This is in part because, in clinical trials, a patient's medical history is routinely coded and reported using the Medical Dictionary for Regulatory Activities (MedDRA) system and is not available in the form of International Classification of Diseases, ninth revision (ICD-9) diagnosis codes which are used in many existing comorbidity indices to determine a patient's comorbidity score. For example, one of the most commonly used indices, the Charlson Comorbidity Index [1], was developed based on hospital records and adapted for use with ICD-9 diagnosis codes [2]. Published indices that provide information on comorbidities without the need for ICD-9 diagnoses can therefore be useful to derive a comorbidity score for clinical trial patients. However, currently, a comorbidity index that can be used with MedDRA terms is not available.

Previously, Sangha et al. developed a novel selfadministered questionnaire to assess comorbid conditions in a clinical setting where medical records are unavailable [3]. Similarly, Wolfe et al. developed an index to assess comorbidities associated with health-related quality of life in four rheumatic diseases using self-report questionnaires [4]. Although both indices are based in rheumatology, each uses patient survey data versus chart-based data to determine comorbidity burden. Considering the methodologies used for these indices, we adapted the Sangha comorbidity index and Wolfe comorbidity index for application to clinical trial data.

The FREEDOM clinical trial was a large phase 3, placebocontrolled study in postmenopausal women with osteoporosis [5]. As patients' medical histories were coded using MedDRA, this trial provided an opportunity to apply the Sangha and Wolfe indices for the purpose of identifying each subjects' comorbidities in the absence of ICD-9 diagnosis codes, and to report on the comorbidity burden of the population studied.

Here, we present the results from the adapted Sangha and Wolfe comorbidity indices that were applied to the FREEDOM clinical trial evaluating postmenopausal women with osteoporosis.

\section{Materials and methods}

\section{Study design}

This was a retrospective analysis of data from the FREEDOM trial. A systematic approach for incorporating input from a panel of clinicians was used to map medical history data in the trial to published indices in order to calculate the comorbidity score for each subject in the trial.

\section{Data source}

The FREEDOM trial design (ClinicalTrials.gov: NCT00089791) has been described previously [5]. FREE
DOM was a 3-year, phase 3, international, randomized, double-blind, placebo-controlled study. Postmenopausal women who enrolled were 60 to 90 years old and had a bone mineral density (BMD) T-score of $<-2.5$ at the lumbar spine or total hip, and $\geq-4.0$ at both sites. Participants received placebo or $60 \mathrm{mg}$ denosumab (Prolia ${ }^{\circledR}$; Amgen Inc., Thousand Oaks, CA, USA) subcutaneously every 6 months for 3 years and were instructed to take calcium $(\geq 1 \mathrm{~g})$ and vitamin $\mathrm{D}$ ( $\geq 400$ IU) daily.

\section{Identifying and mapping comorbid conditions in the FREEDOM trial}

Medical histories of subjects in the FREEDOM trial were coded using MedDRA (version 11.0) standardized international terminology. Briefly, physicians at study sites reported medical histories that were coded using MedDRA preferred terms, which are distinct descriptors for individual medical concepts that are coded from terms that reflect how an observation may be reported in practice. Related preferred terms are further grouped according to a pre-specified hierarchy. The highest level of organization is composed of 26 system organ classes (e.g., gastrointestinal disorders, infections and infestations, musculoskeletal and connective disorders).

Using a pre-specified strategy (Supplementary Fig. S1), a clinician panel of four physicians (three internal and one external to Amgen Inc.) who specialized in cardiology, internal medicine, and/or rheumatology mapped selected MedDRA preferred terms to the comorbid conditions included in the adapted Sangha and Wolfe indices (Table 1). The following steps were conducted to arrive at the comorbidity score for each subject: (1) For each specified comorbid condition, the clinical panelists independently reviewed the MedDRA preferred terms within the respective system organ class for the medical history data collected from all FREEDOM participants; (2) the preferred terms were then classified and used to define a specified comorbid condition. In this study, a comorbid condition was defined as an illness that required medical treatment, health resource utilization, or affected the administration of medication; (3) responses from the panel of clinicians on the classification of preferred terms were consolidated and compared; and (4) any preferred term classification that was not agreed upon by all four physicians was considered a classification discrepancy and was discussed and resolved among panel physicians, and if needed, adjudicated by a fifth physician (external to Amgen Inc.) who specialized in internal medicine and geriatric medicine.

\section{Adaptation of the Sangha and Wolfe indices}

Sangha et al. developed a self-administered comorbidity questionnaire to assess comorbid conditions in clinical and health services research where medical records are unavailable [3]. 
The 13 comorbidities assessed included anemia or other blood diseases, back pain, cancer, depression, diabetes, heart disease, high blood pressure, kidney disease, liver disease, lung disease, arthritis, rheumatoid arthritis, and ulcer or stomach disease. In the adapted Sangha comorbidity index (range 012), 12 of the 13 original comorbidities were evaluated; back pain was not included as a comorbid condition of interest because it was related to the primary outcome of fracture in the FREEDOM trial. As the original Sangha index was not weighted, 1 point was assigned for each condition in the adapted index (Table 1).

Wolfe et al. developed an index to describe comorbidities with four rheumatic diseases through self-reported questionnaires [4]. The original Wolfe index (range 0-9) was composed of 11 present or past comorbid conditions including pulmonary disorders; myocardial infarction (MI); stroke; other cardiovascular (CV) disorders; hypertension; diabetes; spine, hip, and leg fractures; depression; gastrointestinal disorders; gastrointestinal

Table 1 Comorbid conditions in Sangha et al. and Wolfe et al., and weight assignment for consideration in the FREEDOM trial

Weight assignment

$\begin{array}{ll}\text { Sangha et al. } & 1 \\ \text { Anemia or other blood disease } & \\ \text { Back pain }^{\mathrm{a}} & 1 \\ \text { Cancer } & 1 \\ \text { Depression } & 1 \\ \text { Diabetes } & 1 \\ \text { Heart disease } & 1 \\ \text { High blood pressure } & 1 \\ \text { Kidney disease } & 1 \\ \text { Liver disease } & 1 \\ \text { Lung disease } & 1 \\ \text { Osteoarthritis, degenerative arthritis } & 1 \\ \text { Rheumatoid arthritis } & 1 \\ \text { Ulcer or stomach disease } & 2 \\ \text { Wolfe et al. } & 2 \\ \text { Pulmonary disorders } & 1 \\ \text { CV conditions (including MI, stroke, } & 1 \\ \text { and other CV disorders, but } & \\ \text { excluding hypertension) } & \text { Hypertension } \\ \text { Diabetes } & - \\ \text { Spine/hip/leg fracture } & \\ \text { Depression } & \\ \text { Gastrointestinal disorders (including ulcer) } & 1 \\ \text { Cancer } & 1\end{array}$

$C V$ cardiovascular, $M I$ myocardial infarction

${ }^{a}$ Diseases not included in the index as they were related to the primary FREEDOM trial outcome of fracture

${ }^{\mathrm{b}}$ Only included in the index when no other $\mathrm{CV}$ conditions were included ulcer; and cancer. These 11 conditions were then grouped together and differentially weighted, such that a total of 8 conditions were evaluated as follows: 2 points were assigned to pulmonary disorders; 2 points were assigned to $\mathrm{CV}$ conditions (including $\mathrm{MI}$, stroke, and other $\mathrm{CV}$ disorders); and 1 point was assigned to all other conditions (diabetes; spine, hip, and leg fractures; depression; gastrointestinal disorders/ gastrointestinal ulcer; and cancer). In addition, hypertension was assigned a score of 1 point but only in the absence of any $\mathrm{CV}$ conditions. The adapted Wolfe comorbidity index was composed of the same, similarly grouped and weighted comorbid conditions; however, spine, hip, or leg fractures were not included as comorbid conditions of interest because they were related to the primary outcome of fracture in the FREEDOM trial (Table 1). Therefore, a total of 7 conditions were evaluated in the adapted Wolfe index with a range of $0-8$.

\section{Associations between the comorbidity index and subject characteristics and mortality}

Spearman correlations were used to examine associations between the derived comorbidity indices and baseline subject characteristics, including subjects' health-related quality of life status which was assessed using the Osteoporosis Assessment Questionnaire short version (OPAQ-SV) [6] physical function, emotional status, and back pain dimensions, and the EuroQol-5 Dimensions (EQ-5D) [7] utility index with items measuring pain, mobility, usual activities, selfcare, and anxiety/depression. The EQ-5D also includes a visual analog scale (VAS) score for rating overall health status. The association between the comorbidity index and mortality was assessed based on the hazard ratio of comorbidity index as a covariate in a Cox proportional hazard model evaluating mortality. A $P$ value $<0.05$ was considered statistically significant.

\section{Results}

The baseline characteristics of the 7808 women enrolled in the FREEDOM pivotal trial are shown in Table 2. The mean age of the participants was 72.3 years, and $45 \%$ had a fragility fracture at baseline (30\% nonvertebral; $24 \%$ vertebral). Of these subjects, $73.8 \%$ had $\geq 1$ comorbidities based on the adapted Sangha comorbidity index and $70.8 \%$ had $\geq 1$ comorbidities based on the adapted Wolfe comorbidity index. The mean (standard deviation) adapted Sangha comorbidity index was 1.4 (1.2) and the mean (standard deviation) adapted Wolfe comorbidity index was 1.4 (1.3), for all subjects (Table 3).

Based on the adapted Sangha comorbidity index, $26.2 \%$ of the FREEDOM population had a score of $0 ; 33.7 \%$ had a score of $1 ; 23.5 \%$ had a score of $2 ; 11.5 \%$ had a score of 3 ; $3.8 \%$ had a score of $4 ; 1.0 \%$ had a score of 5 ; and $0.3 \%$ had a 
Table 2 FREEDOM trial subject baseline characteristics

\begin{tabular}{ll}
\hline Characteristic & All subjects $(N=7808)$ \\
\hline $\begin{array}{l}\text { Sex, } n(\%) \\
\quad \text { Female }\end{array}$ & $7808(100.0)$ \\
Ethnicity, $n(\%)$ & \\
$\quad$ White or Caucasian & $7238(92.7)$ \\
Age, years & \\
$\quad$ Mean (SD) & $72.3(5.2)$ \\
Age group, $n(\%)$ & \\
$\quad 60$ to 64 years & $414(5.3)$ \\
$\quad 65$ to 69 years & $1644(21.1)$ \\
70 to 74 years & $3279(42.0)$ \\
$\geq 75$ years & $2471(31.6)$ \\
Years since menopause, mean (SD) & $24.2(7.5)$ \\
Prior fragility fracture, $n(\%)$ & $3484(44.6)$ \\
\hline
\end{tabular}

Percentages are based on the number of subjects randomized $N$ number of subjects randomized, $S D$ standard deviation

score of 6 (Fig. 1). Based on the adapted Wolfe comorbidity index, $29.2 \%$ had a score of $0 ; 29.9 \%$ had a score of 1 ; $23.5 \%$ had a score of $2 ; 10.8 \%$ had a score of $3 ; 4.5 \%$ had a score of $4 ; 1.7 \%$ had a score of $5 ; 0.3 \%$ had a score of 6 ; and $<0.1 \%$ had a score of 7 (Fig. 2).

For subjects in the FREEDOM trial, Spearman correlation showed significant, but modest, correlations between the adapted indices with age, body mass index, and the number of medications at baseline (Table 4). An inverse correlation of health-related quality of life measures (OPAQ, EQ-5D, and EQ-5D VAS) with the adapted comorbidity indices was observed, which reached statistical significance. The Spearman correlation between the two indices was $0.9130(P<0.0001)$.

No statistically significant correlations were found between the indices and the FRAX-calculated 10-year major osteoporotic fracture risk with $\mathrm{BMD}$, nor with the 10-year hip fracture risk without BMD in the calculation. A significant but modest correlation was observed with the 10-year major osteoporotic fracture risk without BMD and the adapted Sangha comorbidity index, but not the adapted Wolfe comorbidity index. A significant but modest correlation was observed with the 10-

Table 3 Distribution of comorbidity indices in the FREEDOM trial

All subjects $(N=7808)$

\begin{tabular}{ll}
\hline Adapted Sangha comorbidity index & \\
Mean (SD) & $1.4(1.2)$ \\
Median (min, max) & $1(0,6)$ \\
Adapted Wolfe comorbidity index & \\
Mean (SD) & $1.4(1.3)$ \\
Median (min, max) & $1(0,7)$ \\
\hline
\end{tabular}

$N$ number of subjects randomized, $S D$ standard deviation

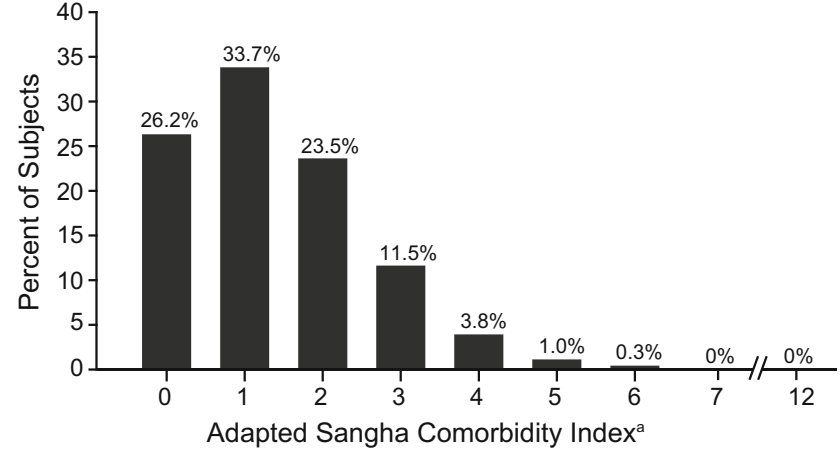

Fig. 1 Distribution of adapted Sangha comorbidity index in the FREE DOM trial. $N=7808{ }^{\text {a }}$ Maximum observed value was 6

year hip fracture risk with BMD and the adapted Wolfe comorbidity index, but not the adapted Sangha comorbidity index (Table 4). In addition, no statistically significant correlations were found between the indices and the number of prevalent vertebral fractures; although, a significant but modest correlation was observed with the number of prior fragility fractures at baseline.

When either adapted comorbidity index was included in the model as a covariate for assessing mortality in the FREE DOM population at 36 months, the hazard ratio of the comorbidity index indicated that the mortality risk in the FREE DOM population increased by 27 or $28 \%$ for each unit increase in the adapted Sangha or Wolfe comorbidity index, respectively (both $P<0.0001$ ).

\section{Discussion}

Comorbidities are important considerations in adjusting for risk of outcomes in clinical trials and are relevant to the interpretation of clinical trial results. However, most comorbidity indices currently available are not designed for use with clinical trial data because they are designed to be applied to secondary databases and rely on ICD-9 codes. Notably, neither the Sangha nor Wolfe comorbidity indices rely on ICD-9

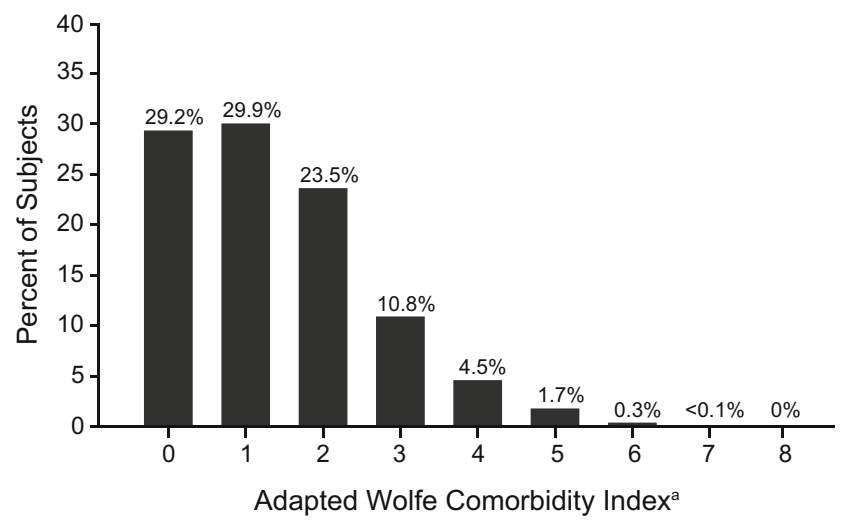

Fig. 2 Distribution of adapted Wolfe comorbidity index in the FREE DOM trial. $N=7808{ }^{a}$ Maximum observed value was 7 
Table 4 Spearman correlation of subject baseline characteristics with adapted Sangha and Wolfe comorbidity indices in the FREEDOM trial

\begin{tabular}{|c|c|c|c|c|}
\hline & \multicolumn{2}{|l|}{ Adapted Sangha comorbidity index ${ }^{\mathrm{a}}$} & \multicolumn{2}{|l|}{ Adapted Wolfe comorbidity index ${ }^{\mathrm{b}}$} \\
\hline & Spearman correlation $(N=7808)$ & $P$ value & Spearman correlation $(N=7808)$ & $P$ value \\
\hline Number of medications taken at baseline & 0.552 & $<0.0001$ & 0.541 & $<0.0001$ \\
\hline \multicolumn{5}{|l|}{ OPAQ dimension } \\
\hline Physical function $(0 \text { to } 100)^{\mathrm{c}}$ & -0.296 & $<0.0001$ & -0.277 & $<0.0001$ \\
\hline Emotional status $(0 \text { to } 100)^{\mathrm{c}}$ & -0.302 & $<0.0001$ & -0.282 & $<0.0001$ \\
\hline Back pain $(0 \text { to } 100)^{\mathrm{C}}$ & -0.243 & $<0.0001$ & -0.222 & $<0.0001$ \\
\hline EQ-5D health state index score $(-0.594 \text { to } 1)^{\mathrm{c}}$ & -0.301 & $<0.0001$ & -0.278 & $<0.0001$ \\
\hline EQ-5D visual analog scale $(0 \text { to } 100)^{c}$ & -0.292 & $<0.0001$ & -0.263 & $<0.0001$ \\
\hline Body mass index $\left(\mathrm{kg} / \mathrm{m}^{2}\right)$ & 0.178 & $<0.0001$ & 0.165 & $<0.0001$ \\
\hline Age (years) & 0.165 & $<0.0001$ & 0.162 & $<0.0001$ \\
\hline 10 -year major osteoporotic fracture risk with $\mathrm{BMD}^{\mathrm{d}}$ & -0.012 & 0.2999 & 0.017 & 0.1353 \\
\hline 10 -year major osteoporotic fracture risk without $\mathrm{BMD}^{\mathrm{d}}$ & -0.025 & 0.0254 & 0.005 & 0.6463 \\
\hline 10-year hip fracture risk with BMD $^{d}$ & -0.001 & 0.9364 & 0.023 & 0.0395 \\
\hline 10-year hip fracture risk without $\mathrm{BMD}^{\mathrm{d}}$ & -0.022 & 0.0537 & 0.005 & 0.6524 \\
\hline Number of prevalent vertebral fractures & -0.0003 & 0.9774 & 0.003 & 0.7975 \\
\hline Number of prior fragility fractures & 0.039 & 0.0006 & 0.046 & $<0.0001$ \\
\hline
\end{tabular}

$N$ number of subjects randomized, $B M D$ bone mineral density, $E Q-5 D$ EuroQol-5 Dimensions, $O P A Q$ osteoporosis assessment questionnaire

${ }^{a}$ Back pain was excluded from the calculation because it was related to the primary outcome of fracture in the FREEDOM trial

${ }^{\mathrm{b}}$ Fracture history was excluded from the calculation because FREEDOM was a fracture study

${ }^{\mathrm{c}} \mathrm{A}$ higher score indicates a more preferred health status

${ }^{\mathrm{d}}$ Based on FRAX version 3.1

codes and therefore may not be directly applied for use with clinical trial data.

In this report, the Sangha and Wolfe comorbidity indices were adapted and applied to FREEDOM clinical trial data to assess the comorbidity burden in this subject population of postmenopausal women with osteoporosis. In doing so, we found a robust correspondence between the two indices. The comorbidity index for the FREEDOM trial, as assessed by the adapted indices, ranged between 0 and 7; however, the majority of subjects ( $60 \%)$ had fewer than two comorbid conditions given the trial eligibility criteria.

Based on the methodology used here, these adapted indices could be used to evaluate other disease states as well. Depending on the trial population of interest, the Wolfe index offers some potential advantages over the Sangha index. The Wolfe index allows for the weighting of disorders and more serious conditions, such as pulmonary and CV disorders, are assigned higher weights. Further, the Wolfe index can potentially include fracture as another comorbid condition. However, as the Wolfe index was originally developed in patients with fibromyalgia, noninflammatory rheumatoid disorders, rheumatoid arthritis, and systemic lupus erythematosus, these conditions were not considered comorbidities and were thus excluded from the index. Subjects with comorbid rheumatoid conditions can therefore only be identified using the Sangha index. Nevertheless, the correlations of the two indices with baseline characteristics were consistent, suggesting that when applied to osteoporosis trial populations, their performance is equivalent (or similar).

Limitations of this study include the use of MedDRA to classify medical diagnoses for the identification of comorbid conditions. Although MedDRA terms are standardized to ensure consistency in use, MedDRA is updated twice annually, which could prove challenging when attempting to compare across clinical trials that have used different versions. In addition, the medical condition review process was dependent on the reviewer's opinion and subject to interpretation of the data. Although consensus agreement had to be reached by the panel members, a different panel of physicians may make a different comorbid condition determination.

The approach proposed in this study was also limited by the medical history information collected or reported in the case report form (CRF). Pre-existing medical conditions may or may not be captured on the CRFs in clinical trials. Unlike claims-based analyses, a subject's history cannot be searched for a number of prior years, which may impact the outcome of interest. In addition, while statistically significant, some of the observed correlations between the indices and baseline characteristics were modest (e.g., OPAQ, EQ-5D), suggesting that the indices may not fully account for the variation in baseline health status. Finally, inclusion and exclusion criteria in a clinical trial usually result in the enrollment of 
patients with fewer comorbidities and, consequently, may represent a relatively healthier patient population which may limit the generalizability of study findings to populations with greater baseline morbidity.

Despite these limitations, the application of comorbidity indices to both clinical trials and observational studies could assist in data interpretation and future research questions, such as differences in the frequency and type of comorbidities, as well as comorbidity burden, in patients in populations of interest (e.g., gender, age, geography), both within the trial and across trials. Recent research has shown that there are marked geographic differences in the prevalence of comorbidities [8] and that comorbidity burden may also be associated with or influence clinical outcomes [9]. For secondary analyses of trial data that are aimed at answering research questions that require adjustment of subjects' comorbidity status, the adaptation of the Wolfe and Sangha indices offers an approach to determine the comorbidity index of a subject in the absence of ICD-9 diagnosis codes. Additional studies are needed to determine if these methodologies, when applied to claims data, result in comparable identification of patients' comorbidity status and may serve as a potential validation of the adapted indices. However, of note, our evaluation of mortality during the FREEDOM trial using the adapted Wolfe and Sangha indices to adjust for comorbidity showed that there was a significant positive correlation between comorbidity and mortality. These data demonstrate that these adapted indices work well as predictors of mortality.

In conclusion, we adapted two existing comorbidity indices to evaluate and characterize an osteoporosis trial population, applied them to the FREEDOM phase 3 clinical trial, and found a high correlation between the two adapted indices. These data suggest that it is possible to adapt existing comorbidity indices to assess comorbidity burden in other disease states and randomized clinical trials. Application of these indices to clinical trials would allow for a more comprehensive description of the trial population and provide a means for more complete interpretation of clinical trial results and supplemental secondary analyses of clinical trial data that require adjustment for comorbidities.

Acknowledgments This study was funded by Amgen Inc. and Lisa Humphries, Ph.D. of Amgen Inc. provided the medical writing assistance.

Conflicts of interest S.L. Silverman received research grants from Amgen Inc. and is a speaker's bureau member, consultant, and national advisory board member for Amgen Inc. A. Wang, L. Cheng, Y. Yang, M. Geller, A. Grauer, and H.N. Viswanathan are employees of Amgen Inc. and hold stock/stock options from Amgen Inc. C. Libanati is an employee of UCB Pharma, is a former employee of Amgen Inc., and holds stock/ stock options from Amgen Inc. D. Revicki received research grants from Amgen Inc. and is a consultant for Amgen Inc. M. Nevitt has nothing to disclose.

Open Access This article is distributed under the terms of the Creative Commons Attribution-NonCommercial 4.0 International License (http:// creativecommons.org/licenses/by-nc/4.0/), which permits any noncommercial use, distribution, and reproduction in any medium, provided you give appropriate credit to the original author(s) and the source, provide a link to the Creative Commons license, and indicate if changes were made.

\section{References}

1. Charlson ME, Pompei P, Ales KL, MacKenzie CR (1987) A new method of classifying prognostic comorbidity in longitudinal studies: development and validation. J Chronic Dis 40:373-383

2. Deyo RA, Cherkin DC, Ciol MA (1992) Adapting a clinical comorbidity index for use with ICD-9-CM administrative databases. J Clin Epidemiol 45:613-619

3. Sangha O, Stucki G, Liang MH, Fossel AH, Katz JN (2003) The selfadministered comorbidity questionnaire: a new method to assess comorbidity for clinical and health services research. Arthritis Rheum 49:156-163

4. Wolfe F, Michaud K, Li T, Katz RS (2010) Chronic conditions and health problems in rheumatic diseases: comparisons with rheumatoid arthritis, noninflammatory rheumatic disorders, systemic lupus erythematosus, and fibromyalgia. J Rheumatol 37:305-315

5. Cummings SR, San Martin J, McClung MR, Siris ES, Eastell R, Reid IR, Delmas P et al (2009) Denosumab for prevention of fractures in postmenopausal women with osteoporosis. N Engl J Med 361:756-765

6. Silverman SL (2000) The osteoporosis assessment questionnaire (OPAQ): a reliable and valid disease-targeted measure of healthrelated quality of life (HRQOL) in osteoporosis. Qual Life Res 9: 767-774

7. EuroQol G (1990) EuroQol — a new facility for the measurement of health-related quality of life. Health Policy 16:199-208

8. Dougados M, Soubrier M, Antunez A, Balint P, Balsa A, Buch MH, Casado G et al (2014) Prevalence of comorbidities in rheumatoid arthritis and evaluation of their monitoring: results of an international, cross-sectional study (COMORA). Ann Rheum Dis 73:62-68

9. Diez-Perez A, Adachi JD, Adami S, Anderson FA Jr, Boonen S, Chapurlat R, Compston JE et al (2014) Risk factors for treatment failure with antiosteoporosis medication: the Global Longitudinal Study of Osteoporosis in Women (glow). J Bone Miner Res 29: 260-267 